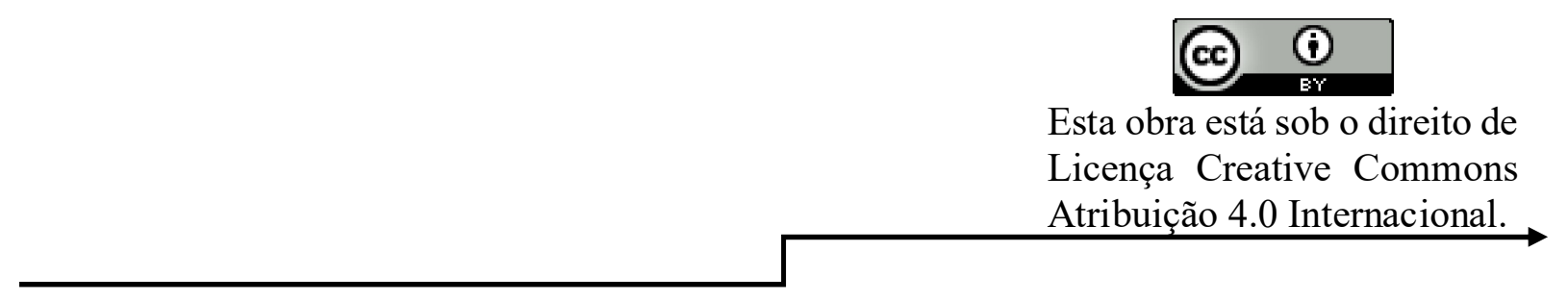

\title{
BIOIMPEDÂNCIA APLICADA À AVALIAÇÃO NUTRICIONAL
}

\author{
Andrea Marques Vanderlei Fregadolli ${ }^{1}$ \\ Fábio Luiz Fregadolli ${ }^{2}$ \\ Augusto Cesar Almeida Barbosa Filho ${ }^{3}$ \\ Edjane Vieira Pires ${ }^{4}$
}

\section{RESUMO}

Para que o levantamento de trabalhos acadêmicos, a respeito de qualquer temática seja rápido, acessível e completo é necessário usar estratégias para direcionar as buscas. Dentre as estratégias mais comuns estão: o uso de aspas em politermos, operador booleano AND, descritor estruturado (com registro no DeCS, Mesh, Thesaurus) e filtros nas bibliotecas virtuais. Essa revisão sistemática tem por objetivo obter um levantamento de publicações científicas sobre avaliação nutricional por meio da bioimpedância na BVS usando descritores livre, estruturado e seus sinônimos. Trata-se de um estudo exploratório, descritivo, bibliográfico, quantitativo-qualitativo, com o universo de publicações visualizadas no filtro da Biblioteca Virtual da Saúde (BVS), por meio de descritores livre "Bioimpedância" e estruturado "Impedância elétrica", bem como seus politermos sinônimos (Resistência Ôhmica, Impedância Bioelétrica), com identificador único (DeCS), sob o código D017097. Inicialmente foi utilizado o filtro "Assunto Principal" do tipo "Avaliação Nutricional" da BVS para refinamento da busca. Os critérios de inclusão foram: textos completos e disponibilidade gratuita. As variáveis analisadas foram: descritores (número total de publicações e número de publicações disponíveis), objetivos e conclusões dos 28 artigos científicos obtidos no levantamento das publicações a partir dos quatro descritores citados anteriormente e filtros da BVS (coleções, base de dados, assunto principal, aspecto clínico, limite, país/região como assunto, idioma, revista, assunto da revista, ano de publicação, tipo de documento e país de afiliação). Dos 28 artigos obtidos nas varreduras da BVS, com os quatro descritores, 13 estavam escritos em português, apenas um em espanhol e 14 em inglês. A bioimpedância (único descritor livre) foi a que obteve maior publicação escrita em português $(\mathrm{n}=10)$.

\footnotetext{
${ }^{1}$ Graduada em Farmácia, Educação Física e Análise e Desenvolvimento de Sistemas. Mestre em Modelagem Computacional de Conhecimento pele Universidade Federal de Alagoas (UFAL, 2011). Doutora em Química e Biotecnologia (UFAL).

2 Possui graduação em Zootecnia pela Universidade Estadual de Maringá (1996), mestrado em Zootecnia pela Universidade Estadual de Maringá (2000) e doutorado em Zootecnia pela Universidade Estadual Paulista Júlio de Mesquita Filho (2004).

3 Possui ensino-médio-segundo-grau pelo Colégio Pontua I(2018).

4 Possui graduação em Química Licenciatura pela Universidade Federal de Alagoas (2005), mestrado em Química e Biotecnologia pela Universidade Federal de Alagoas (2008) e doutorado em Química e Biotecnologia pela Universidade Federal de Alagoas (2013).
} 
Como esse descritor não é codificado, não possui identificador único, justificando seu baixo potencial indexador. Contudo, os resultados apontam a necessidade de inserir no cadastro de registro DeCS/MeSH, uma vez que foi o descritor com menos "lixo", ou seja, um número total de publicações bem inferior aos demais e um quantitativo de publicações disponíveis muito próximo aos demais descritores analisados, caracterizando uma importante terminologia.

Palavras-chave: Impedância elétrica. Impedância bioelétrica. Resistência ôhmica. Descritores em ciências da saúde. 


\section{INTRODUÇÃO}

A análise da composição corporal é parte essencial dos programas de emagrecimento e de condicionamento físico. A bioimpedância elétrica é uma técnica que contribui na profilaxia e tratamento de diversas condições patológicas, dente elas: insuficiência renal, hepatopatias, neoplasias, diabetes, hipertensão arterial, dislipidemias e cardiopatias. Os equipamentos de bioimpedância são modernos, possuem tipos e fabricantes variados, de maneira que os mais avançados usam tecnologia para analisar a composição corporal de: água intra/extracelular e corporal total, proteína, minerais, massa muscular esquelética e de gordura, estimativa de gordura segmentar, Índice de edema corporal e segmentar, taxa metabólica basal, relação cintura-quadril, área de gordura visceral, grau de obesidade, conteúdo mineral ósseo, ângulo de fase corporal total e segmentar.

O estado nutricional de uma pessoa pode ser estimado por equipamentos que determinam o fracionamento da composição corporal, dentre eles a bioimpedanciometria. Esse exame é viável para a prática clínica e para estudos epidemiológicos, pois é portátil, de fácil manuseio, não é invasivo e permite uma quantidade satisfatória de reprodução. Contudo, a bioimpedância elétrica é uma área carente de pesquisas nacionais necessitando de maiores comparações com outros métodos de avaliação nutricional e composição corporal (EICKEMBERG et al, 2011), para validar sua eficácia em relação a outros procedimentos clínicos mais baratos e acessíveis, tais como a antropometria.

A bioimpedância é um método clínico para estimar a composição corporal e o estado nutricional de indivíduos saudáveis ou em situação patológica. Nesse contexto, Guedes (2013, p. 114) afirma que "os procedimentos clínicos são menos dispendiosos, menos rigorosos e de interpretação mais imediata; portanto, de maior aplicação prática", quando comparados com os procedimentos laboratoriais. O mesmo autor garante que os erros de estimativa, quando produzidos pela impedância elétrica, possuem limites aceitáveis.

A bioimpedância é capaz de estimar os parâmetros apontados no primeiro parágrafo, desse trabalho, a partir da análise do fluxo da corrente elétrica. Esse tipo de exame é largamente aceito e o valor dos equipamentos podem variar de $\mathrm{R} \$ 8.000,00$ a R\$92.000,00. Contudo, quando comparado com a pletismografia (Bod $\operatorname{Pod}\left({ }^{\circledR}\right)$ e a absortometria radiológica de dupla energia (DXA) ainda é considerado de baixo custo (MELLO et al, 2005; EICKEMBERG et al, 2011). O fluxo da corrente elétrica no ser humano depende do tipo de tecido, componentes e fluidos corporais, por isso possui taxas diferentes distribuídas pelo corpo, 
sendo esse o princípio da impedância elétrica. Uma resistência maior à corrente elétrica pode ser observada no tecido adiposo quando comparado com o tecido muscular (COCETTI; CASTILHO; BARROS; 2009; EICKEMBERG et al, 2011; FUJIOKA, 2012; MORAIS et al, 2013).

Apesar de a temática principal ser: "Avaliação nutricional por meio da bioimpedância"; o público de interesse nesse estudo compreende não apenas os profissionais da área da saúde/humano-biológica (nutricionistas, médicos, biólogos, fisioterapeutas, educadores físicos) e exata (tecnologias), bem como professores, acadêmicos da graduação e pós-graduação, pesquisadores e profissionais bibliotecários, no sentido de facilitar a localização da terminologia adequada e reconhecida mundialmente para descrever, organizar e ajudar a encontrar a informação que procura, incentivando autonomia do usuário na recuperação da informação (PELLIZZON, 2004).

Novos equipamentos foram lançados recentemente e os que fazem avaliações mais completas e precisas são relativamente caros, pois são importados. Mediante esses fatos, espera-se encontrar pouca informação científica sobre esse tipo de exame. Por isso, é necessário usar estratégias de busca por meio de descritor para obtenção de documentos científicos que abordem essa temática no âmbito mundial.

Acredita-se que estratégias de busca podem ser utilizadas para obter publicações sobre avaliação nutricional por meio da bioimpedância, nesse contexto surgiu a seguinte pergunta que norteou esse estudo: Quais descritores (livre e estruturado) e filtros são mais eficazes em varreduras realizadas na Biblioteca Virtual da Saúde para obtenção de trabalhos acadêmicos que tratam sobre bioimpedância na avaliação nutricional?

O presente trabalho tem como objetivo integrar as informações presentes nas publicações científicas sobre avaliação nutricional por meio da bioimpedância na BVS usando descritores livre, estruturado e seus sinônimos. Mas, para atingir tal objetivo é necessário usar estratégias de busca por meio de descritores em ciências da saúde que direcionem as varreduras para bioimpedância na avaliação nutricional, analisar o conteúdo (objetivos e conclusões) das publicações científicas da Biblioteca Virtual da Saúde (BVS) visualizadas em varreduras obtidas com os descritores sugeridos e mencionar a importância da BVS e de seus filtros na busca de trabalhos acadêmicos sobre avaliação nutricional por meio da bioimpedância. 


\section{METODOLOGIA}

Trata-se de uma revisão bibliográfica sistemática integrativa, que se caracteriza por sumarizar e analisar o conhecimento científico já produzido sobre o tema investigado, permitindo conclusões que articulam os resultados obtidos em diferentes estudos (GANONG, 1987; BOTELHO; CUNHA; MACEDO, 2011).

A literatura nacional e internacional consultada foram pautadas em seis etapas distintas e sequenciais, a saber: $1^{\text {a }}$ Etapa: identificação do tema e seleção da questão de pesquisa, por meio da definição do problema, formulação de uma pergunta norteadora e escolha da estratégia de busca, descritores e bases de dados mais eficazes no levantamento das publicações; $2^{\mathrm{a}}$ Etapa: estabelecimento dos critérios de inclusão e exclusão; $3^{\text {a }}$ Etapa: Identificação dos estudos pré-selecionados e selecionados através da leitura dos agentes indexadores das publicações, como resumo, palavras-chave e título, bem como organização dos estudos pré-selecionados e identificação dos estudos selecionados; $4^{\mathrm{a}}$ Etapa: Categorização dos estudos selecionados, com elaboração e uso da matriz de síntese, além de análise das informações, formação de uma biblioteca individual e avaliação crítica dos estudos selecio-

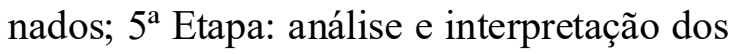
resultados permitindo discuti-los; $6^{\mathrm{a}}$ Etapa: apresentação da revisão/síntese do conhecimento a partir da criação de um documento que a descrevesse detalhadamente, o qual contempla propostas para estudos futuros (BOTELHO; CUNHA; MACEDO, 2011; SCHMOELLER et al., 2011).

O levantamento bibliográfico foi realizado por meio de consulta ao portal da Biblioteca Virtual de Saúde (BVS) incluindo as fontes de informações, que compõe a rede BVS: Literatura Latino-Americana e do Caribe em Ciências da Saúde (LILACS), Medical Literature Analysis and Retrieval System Online (MEDLINE), Scientific Electronic Library Online (SciELO), recursos educacionais abertos e sites de internet.

Como estratégia de busca, no sentido de obter estudos relacionados com a temática em estudo, foram iniciadas as varreduras na BVS, em 03 de fevereiro de 2017, por meio de descritores (Quadro 1) livre Bioimpedância e estruturado "Impedância elétrica", bem como seus politermos sinônimos ("Resistência Ôhmica", "Impedância Bioelétrica"), com identificador único (DeCS), sob o código D017097. Inicialmente foi utilizado o filtro "Assunto Principal" do tipo "Avaliação Nutricional" da BVS para refinamento da busca. Para constituir a amostra foram selecionados os trabalhos que atenderam aos seguintes critérios: tipo de documento artigo científico, textos completos disponíveis online, tempo 
de busca sem limitação, publicação no períseguinte temática: avaliação nutricional por odo de 2006 a 2017, que versavam sobre a meio da bioimpedância.

Quadro 1 - Terminologias usadas no levantamento de publicação sobre avaliação nutricional por meio da bioimpedância na Biblioteca Virtual de Saúde.

\begin{tabular}{|c|c|c|c|}
\hline $\mathbf{N}^{\mathbf{0}}$ & Descritor & Tipo de Terminologia & ID \\
\hline 1 & Bioimpedância & Livre (ausente do DeCS, MeSH, Thesaurus) & $* * *$ \\
\hline 2 & "Impedância elétrica" & Estruturado - DeCS & D017097 \\
\hline 3 & "Impedância bioelétrica" & Sinônimo de Impedância elétrica - DeCS & $* * *$ \\
\hline 4 & "Resistência ôhmica" & Sinônimo de Impedância elétrica - DeCS & $* * *$ \\
\hline
\end{tabular}

Fonte: DECS, 2017; MESH, 2017; THESAURUS, 2017.

Após aplicação dos filtros e leitura dos títulos, palavras-chave e resumos, foi excluída toda publicação duplicada e as que não correspondiam aos critérios de inclusão, totalizando 28 artigos científicos, os quais foram submetidos à revisão integrativa, tratando e interpretando as informações, sistematizando-as e categorizando-as.

As publicações obtidas no Portal BVS foram perfilizadas, por meio das vari-

\section{RESULTADOS}

A aquisição de documentos científicos que atendam ao objeto de estudo traçado depende da escolha correta dos descritores, das bases de busca e de seus filtros (metadados). áveis: descritores (número total de publicações e número de publicações disponíveis) e filtros da BVS (coleções, base de dados, assunto principal, aspecto clínico, limite e idioma). Realizou-se uma análise estatística não paramétrica por meio do teste qui-quadrado, no software PAleontological STatistics (Past - Version 2.16) (BENTON, 1994), para observar a significância da variável descritores.

\section{Perfil das publicações}

Segue, abaixo, Tabela 1, com os dados dos descritores utilizados nas varreduras realizadas na Biblioteca virtual da saúde (BVS), com a finalidade de obter textos completos de publicações científicas sobre bioimpedância aplicada na avaliação nutricional. 
Tabela 1 - Total de publicações e textos disponíveis na Biblioteca Virtual de Saúde usando descritores estruturados.

\begin{tabular}{l|c|c|c|c}
\hline \multicolumn{1}{c|}{ Descritor } & $\begin{array}{c}\text { Total de publi- } \\
\text { cações sem o fil- } \\
\text { tro “Assunto } \\
\text { principal" }\end{array}$ & $\begin{array}{c}\text { Total de publi- } \\
\text { cações com o filtro } \\
\text { “Assunto princi- } \\
\text { pal”, do tipo: Ava- } \\
\text { liação Nutricional }\end{array}$ & $\begin{array}{c}\text { Textos } \\
\text { completos } \\
\text { disponíveis }\end{array}$ & $\begin{array}{c}\text { Textos apro- } \\
\text { veitados na } \\
\text { pesquisa }\end{array}$ \\
\hline Bioimpedância & 400 & 288 & 27 & 13 \\
\hline "Impedância elétrica" & 14.296 & 121 & 57 & 15 \\
\hline "Impedância bioelé- & 14.310 & 123 & 59 & 17 \\
trica" & 14.258 & 120 & 56 & 14 \\
\hline "Resistência ôhmica" & & & & \\
\hline
\end{tabular}

Fonte: BVS, 2017.

O descritor livre bioimpedância foi o que obteve menor número de publicações na BVS, com 13 artigos disponíveis e o descritor "impediância bioelétrica" (sinônimo de "impedância elétrica", no DeCS) foi o que possuiu maior número de publicações disponíveis, com 17 artigos científicos. Apesar da varredura realizada com a terminologia bioimpedância ter sido menor quando comparado com os outros descritores, não houve diferença significativa $\left(\mathrm{X}^{2}=\right.$ 2,61 ns) no teste qui-quadrado.

Como a produção científica do assunto principal "Avaliação nutricional” dos descritores bioimpedância," impedância elétrica", "impedância bioelétrica" e "resistência ôhmica", pode ser estimada e refinada na BVS. Então, nessa pesquisa, foram analisados 12 metadados (filtros para refinar a busca de dados), do descritor bioimpedância, disponíveis na BVS, do tipo: coleções, base de dados, assunto principal, aspecto clínico, limite e idioma.
Após varredura realizada com o descritor livre bioimpedância na BVS foi possível observar que as bases de dados internacionais possuíam mais publicações que as nacionais. A base de dados LILACS teve mais artigos científicos que o IBECS, Coleciona SUS, BDENF-Enfermagem, Index Psicologia e MEDLINE.

Os assuntos principais que obtiveram mais de três publicações foram avaliação nutricional ( $n=37)$, composição corporal $(n=14)$, estado nutricional $(n=13)$, impedância elétrica $(n=8)$, antropometria $(n=4)$, desnutrição $(n=4)$, neoplasias $(n=3)$ e obesidade $(n=3)$. $O$ aspecto clínico com mais publicações foi prognóstico $(n=36)$, seguido do diagnóstico $(n=16)$, etiologia $(n=9)$ e predição $(n=2)$.

Os limites com mais de três artigos científicos foram humanos $(n=31)$, feminino $(n=17)$, masculino $(n=17)$, adulto $(n=8)$, idoso $(n=5)$, criança $(n=4)$ e adolescente $(n=3)$. O idioma com mais publicação foi o português $(n=20)$, seguido do espanhol $(\mathrm{n}=11)$ e por último inglês $(\mathrm{n}=8)$. 


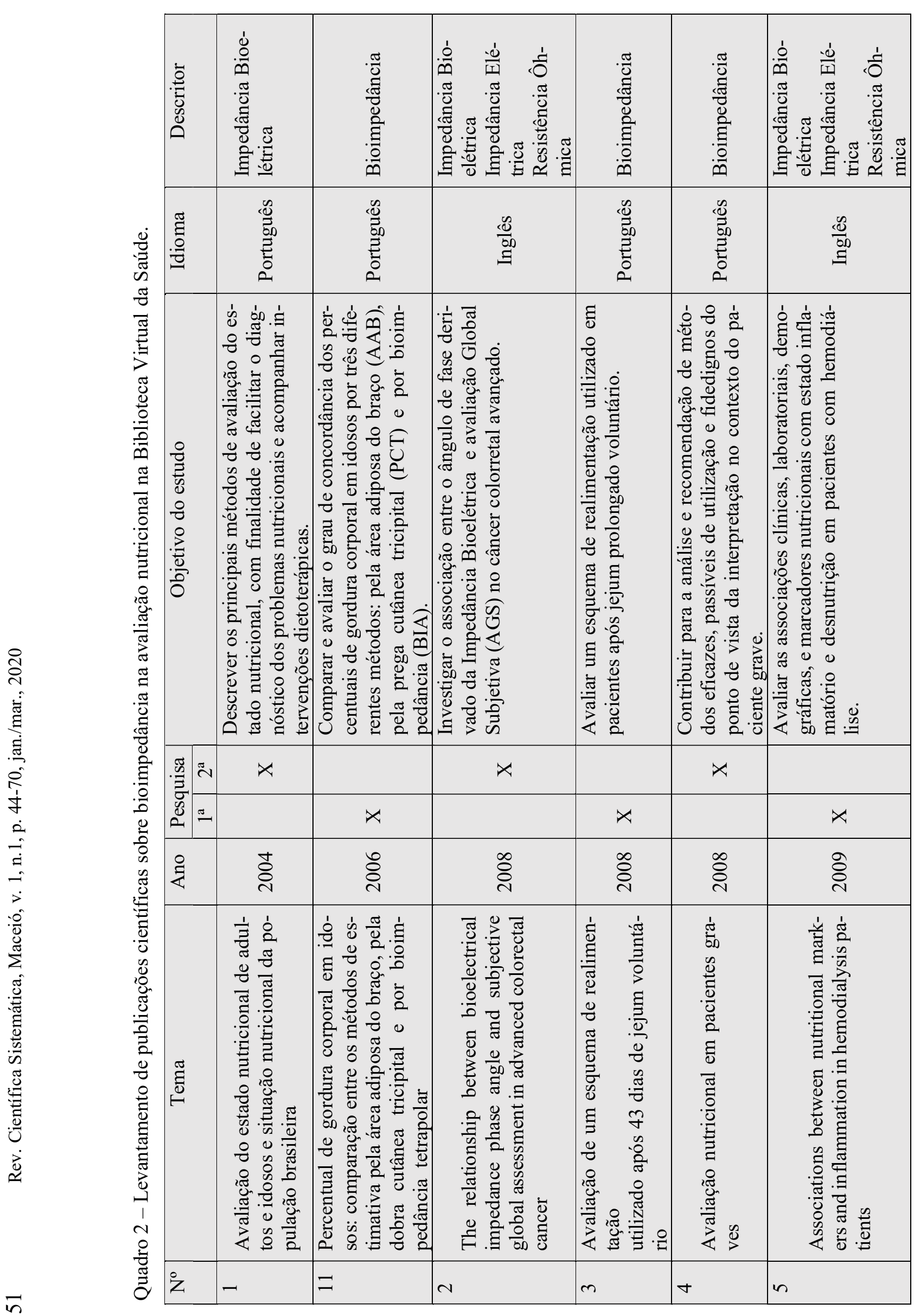




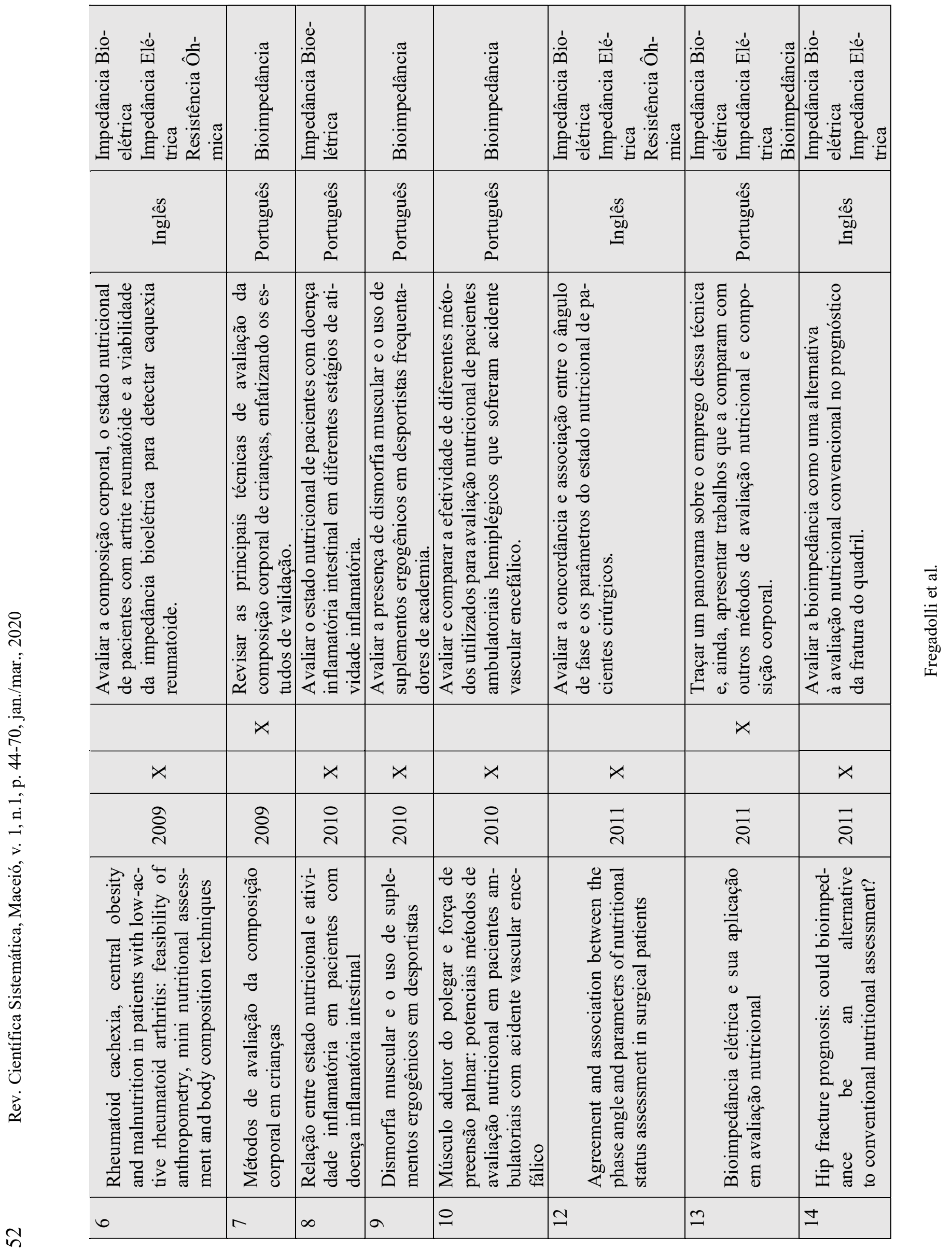




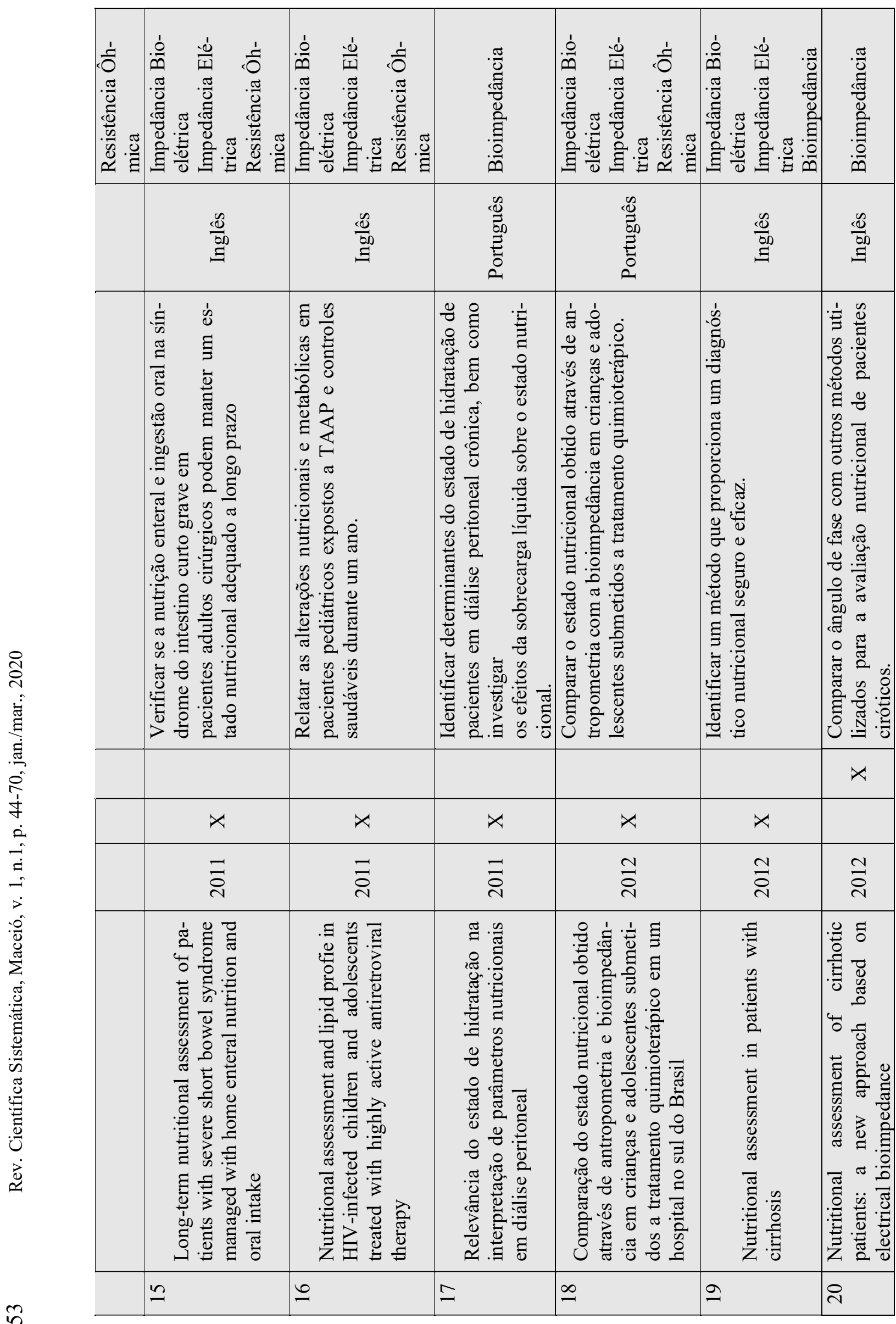

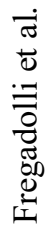




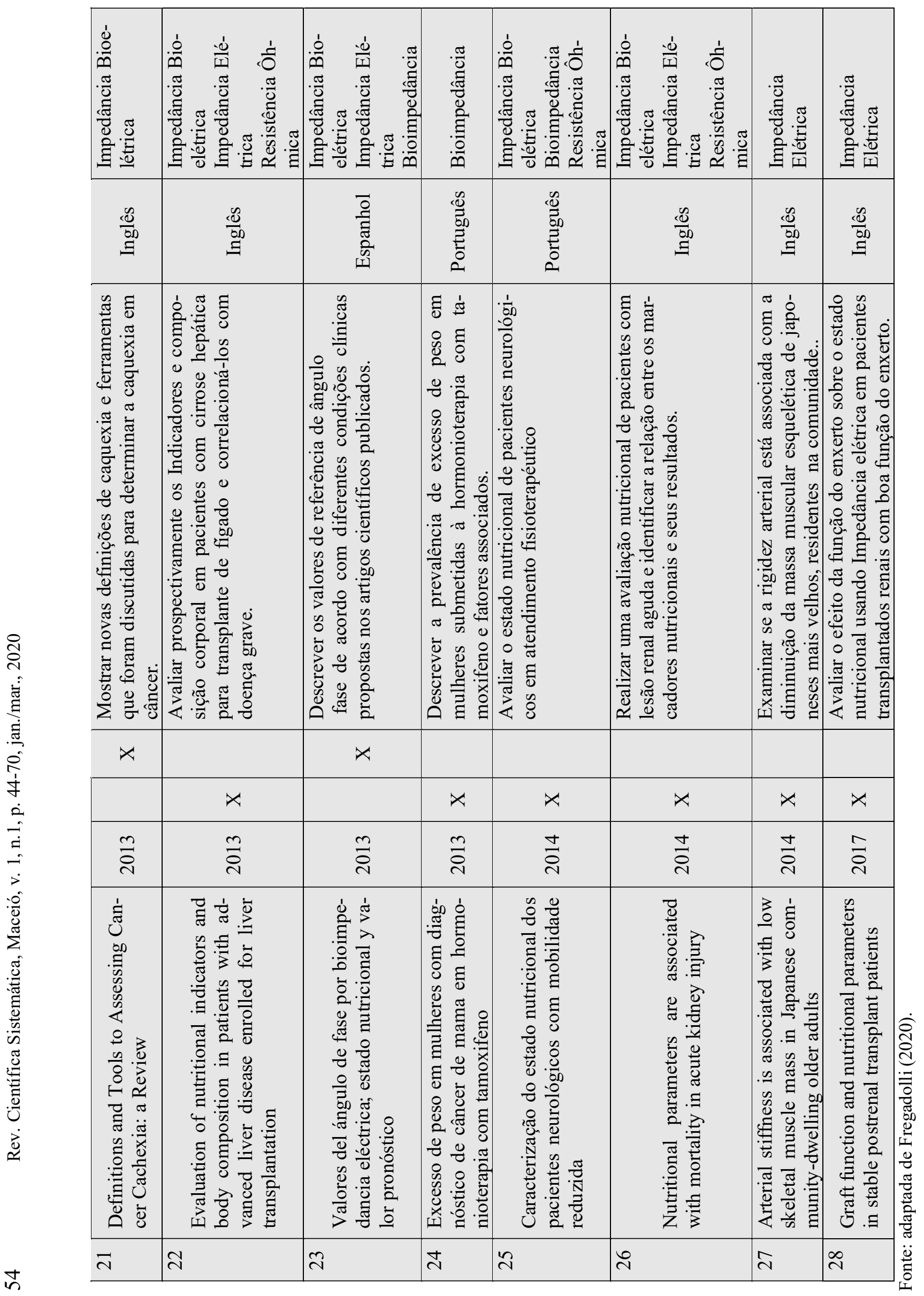




\section{Revisão integrativa}

A partir da análise da amostra desta pesquisa, foi possível compreender a bioimpedância na avaliação nutricional de pacientes, através das seguintes categorias: (1) Diferentes grupos etários com ou sem fratura no quadril; (2) Diminuição muscular esquelética em idosos e

\section{DISCUSSÃO}

Seguem, abaixo, as categorias temáticas elaboradas a partir da revisão sistemática integrativa.

\section{Diferentes grupos etários com ou sem fratura no quadril}

A bioimpedância é um método duplamente indireto, rápido, sensível, reprodutível, barato e não invasivo, que avalia o dano celular e a composição corporal, como, o grau de concordância dos percentuais de gordura corporal em diferentes grupos etários. Esse método também é utilizado como marcador de estado nutricional, inclusive no prognóstico da fratura do quadril de pacientes idosos, por isso é mais utilizado em pacientes hospitalizados não aptos para avaliação antropométrica por fratura e imobilidade. Os parâmetros da bioimpedência também podem ser usados para intervenções profiláticas, no acompanhamento do estado nutricional de indi- dismorfia muscular; (3) Cirrose hepática; (4) Farmacoterapias e Neoplasias; (5) Patologias com processo inflamatório; (6) Patologias Neorológicas; (7) Distúrbio no equilíbrio de fluidos; (8) Realimentação no jejum prolongado voluntário e (9) Cirúrgicos.

víduos normais ou com quadro patológico, bem como na realimentação no jejum prolongado voluntário.

Eickemberg et al. (2011) traçou um panorama sobre o emprego da bioimpedância e, ainda, apresentou trabalhos que a comparou com outros métodos de avaliação nutricional e composição corporal. Os estudos não só revelaram uma importante habilidade dessa técnica em determinar o fracionamento dos componentes corporais, mas também valorizaram sua utilização como marcador de estado nutricional e de avaliação de dano celular. Contudo, o mesmo recomendou o uso de critérios mais confiáveis para análise e interpretação dos resultados, enfatizando a prioridade de pesquisas que desenvolvam equações específicas para a população brasileira, inclusive para diferentes grupos etários.

Sant'anna, Priore e Franceschini (2009) revisaram as principais técnicas de avaliação da composição corporal de 
crianças, enfatizando os estudos de validação. Dentre os métodos para avaliação da composição corporal de crianças, os métodos indiretos, do tipo: plestimografia e absortometria radiológica de dupla energia foram os mais utilizados. Já os duplamente indiretos mais utilizados foram o índice de massa corporal, as pregas cutâneas, a bioimpedância, a circunferência da cintura, a relação cintura/ quadril, o índice de conicidade e a relação cintura/estatura. Há vários métodos para avaliar a composição corporal em crianças, cada um com suas vantagens e desvantagens. Por isso, é preciso eleger aquele que melhor detecte o problema que se pretende corrigir, levando em consideração os custos, o nível de treinamento dos avaliadores, o tempo de execução, a receptividade da população e os possíveis riscos à saúde.

Em estudos populacionais, de todas as faixas etárias, a Organização Mundial de Saúde preconiza a utilização do Índice de Massa Corpórea para diagnóstico das desordens nutricionais. Entretanto, a avaliação do estado nutricional não dispõe de padrão-ouro para diagnóstico dessas desordens, pois a escolha do método depende dos objetivos da avaliação. Por exemplo, os índices múltiplos permitem detectar desnutrição subclínica em pacientes hospitalizados com a possibilidade de realizar exames laboratoriais. A avaliação do estado nutricional de idosos pode ser realizada por vários instrumentos, dentre eles, a Miniavaliação Nutricional (MAN), a qual faz uso combinado da antropometria, avaliação dietética, avaliação clínica global e autopercepção de saúde. Outro método é a Bioimpedância, que apesar de pouco utilizado, possui diversas vantagens, por ser rápido, não-invasivo e relativamente barato, sendo mais utilizado em pacientes hospitalizados e inquéritos populacionais (ACUÑA; CRUZ, 2004).

Machado, Coelho e Coelho (2006) compararam e avaliaram o grau de concordância dos percentuais de gordura corporal em idosos por três diferentes métodos: pela área adiposa do braço $(\mathrm{AAB})$, pela prega cutânea tricipital (PCT) e por bioimpedância (BIA). Todos os coeficientes de correlação intraclasse $(\mathrm{CCI})$ entre as variáveis foram estatisticamente significantes. Todos os métodos também apresentaram elevada correlação com as variáveis antropométricas isoladamente. As mulheres apresentaram maior percentual de gordura pelos três métodos. O percentual de gordura estimado pela bioimpedância apresentou melhor correlação com o IMC, que reflete a massa corporal total e com a $\mathrm{CC}$, que reflete o depósito abdominal. O método utilizado influi na estimativa 
do percentual de gordura a partir dos membros superiores. A AAB sobrestima o percentual de gordura com relação à Equação de Siri e BIA, e seu uso como estimador de gordura global necessita de mais estudos.

Schiper et al. (2011) avaliaram a bioimpedância (resistência, reatância e ângulo de fase) como uma alternativa à avaliação nutricional convencional no prognóstico da fratura do quadril de pacientes idosos. Esse estudo, concluiu que as medidas primitivas de bioimpedância, que até então não tinham sido investigadas, estavam prognosticamente relacionadas com a mortalidade precoce e tardia. Esses marcadores de desnutrição relacionada à doença e, especialmente, de reatância devem ser estudados em pacientes não aptos para avaliação antropométrica por fratura e imobilidade.

\section{Diminuição muscular esquelética em idosos e dismorfia muscular}

A bioimpedância é um método seguro para avaliar a massa muscular absoluta do esqueleto, assim como a massa segmentar e massa muscular de gordura. Tais informações podem ser usadas para relacionar o envelhecimento vascular com o declínio da massa muscular, além de relacionar o uso de suplementos e a presença de dismorfia muscular.
Aurélio et al. (2014) por meio da tecnologia multifrequência segmentar direta do equipamento "InBody" realizou o exame de bioimpedância, o qual forneceu valores para massa muscular absoluta do esqueleto, percentual de gordura corporal, massa gorda absoluta e massa muscular segmentar (braços e pernas/direitos e esquerdos, e tronco). A partir destas medições, as massas absolutas do músculo esquelético e de gordura foram ajustadas posteriormente pela altura para determinar Índice de Massa Muscular Esquelética (SMI) e o Índice de Massa Gorda (FMI). Com o objetivo de examinar se a rigidez arterial estava associada com a diminuição da massa muscular esquelética de japoneses mais velhos, residentes na comunidade, concluiu que a rigidez arterial está associada ao baixo Índice de Massa Muscular Esquelética (SMI), mesmo quando ajustado por múltiplos fatores, mostrando uma estreita interação entre o envelhecimento vascular e o declínio da massa muscular.

Lima, Moraes e Kirsten (2010) avaliaram a presença de dismorfia muscular (DM) e o uso de suplementos ergogênicos em desportistas frequentadores de academia. O teste de bioimpedância foi aplicado para avaliar o corpo, sendo possível obter os dados de massa magra e gordura. O risco de DM foi detectado 
com uma escala (MASS- Escala de satisfação de aparência muscular). Resultado superior a 52 pontos, apresentou risco de DM. Além disso, informações sobre o uso de suplementos foram coletadas por meio dum questionário. A análise dos resultados identificou que, dos avaliados, $17,4 \%(n=4)$ apresentaram risco positivo para o desenvolvimento de DM. Entretanto, destes, apenas $25 \%$ declararam fazer uso de suplementos alimentares. Conclui-se que não houve relação entre o uso de suplementos e a presença de dismorfia muscular.

\section{Cirrose hepática}

A avaliação nutricional por meio da bioimpedância permite analisar o ângulo de fase, parâmetro este, ideal para a classificação das condições nutricionais de pacientes cirróticos. Esse método mede parâmetros como resistência (contribuição relativa do fluido) e capacitância (membranas celulares), o qual registra a queda na tensão na corrente aplicada.

A Sociedade Europeia de Nutrição Enteral e Parentérica, em 2006, orientou identificar pacientes com cirrose a partir da Avaliação Global Subjetiva (AGS), Análise Antropométrica e Controle de Mão. Balbino e Silva (2012) na tentativa de identificar um método que proporcionasse um diagnóstico nutricional seguro e eficaz de pacientes cirróticos fizeram uma comparação do ângulo de fase (AF) com outros métodos utilizados. Ao analisar a bioimpedância, consideraram um método sensível, reprodutível, seguro e barato que pode ser usado para determinar o estado nutricional. Tal método mede parâmetros como resistência e capacitância, registrando a queda na tensão na corrente aplicada. A redução da tensão é quantificada geometricamente como a transformação angular da proporção da capacitância para a resistência.

Fernandes et al. (2012) realizou avaliações globais clínicas, antropométricas e subjetivas, por meio de dinamometria e bioimpedância na avaliação nutricional em pacientes com cirrose. Os diferentes métodos apresentaram discrepâncias acentuadas, com a porcentagem de pacientes desnutridos variando de 5,4\% a 68,2\%. Os métodos de Força de Preensão Manual e Bioimpedância mostraram uma variação muito alta, com percentuais de desnutrição de $68,2 \%$ para $34,1 \%$, respectivamente. Esse estudo afirmou que a bioimpedância proporciona um diagnóstico nutricional seguro e eficaz, pois apresentou correlação estatisticamente significativa com o escore de Child-Pugh, o qual sugeriu que a identificação do ângulo de fase de $5,44^{\circ}$ 
é o parâmetro ideal para a classificação das condições nutricionais de pacientes cirróticos. No entanto, são necessários mais estudos para confirmar estes dados, no sentido de expandir o número de casos de pacientes classificados como Child-Pugh C.

Vulcano, Carvalhaes e Bakonyi Neto (2013) avaliaram prospectivamente os indicadores e composição corporal por meio de medidas antropométricas, avaliação global subjetiva (AGS) e indicadores bioquímicos, em pacientes com cirrose hepática para transplante de fígado, no sentido de correlacioná-los com doença grave. Foi medida a resistência e ângulo de fase. O diagnóstico de desnutrição variou de acordo com o método. A avaliação global do estado nutricional mostrou melhor correlação com a gravidade da doença do que com os outros métodos avaliados. A bioimpedância de frequência única para análise de composição corporal em pacientes cirróticos deve ser cautelosamente utilizada. No entanto, os vetores primários parecem ser válidos e promissores na prática clínica.

\section{Farmacoterapia, neoplasia e processo inflamatório}

A bioimpedância é capaz de determinar a caquexia reumatoide e em câncer, o estado nutricional de portadores de câncer colorretal avançado, alterações nutricionais e metabólicas em crianças e adolescentes expostos à terapia antirretroviral, a prevalência de excesso de peso em mulheres diagnosticadas com câncer de mama submetidas à hormonioterapia com tamoxifeno, de pacientes com doença inflamatória intestinal em diferentes estágios de atividade inflamatória e também parece ser um método eficaz para avaliação nutricional e aferição de compartimentos corporais em pacientes pediátricos portadores de neoplasias.

Tremeschin et al. (2011) relataram as alterações nutricionais e metabólicas em crianças e adolescentes expostos a terapia antiretroviral (TARV) de alta potência e controles saudáveis durante um ano. A estimativa da massa livre de gordura e da água corporal total, foi obtida por meio da bioimpedância ao mesmo tempo que o peso foi medido. A circunferência do braço, circunferência da cintura, dobra cutânea subescapular e tríceps cutâneo foram também medidos para refletir massa corporal magra e gordura. A análise da bioimpedância de pacientes pediátricos e hebiátricos foi realizada de acordo com o tipo e tempo de TARV. A bioimpedância foi incapaz de detectar diferenças. Os sujeitos da pes- 
quisa tiveram seus parâmetros nutricionais comprometidos quando comparados com o grupo controle. Indivíduos usando inibidor de protease apresentaram piores níveis séricos de triglicerídeos quando comparados com os saudáveis.

Carraro, Schwartz e Behling (2012) compararam o estado nutricional obtidos através de antropometria usando dados de peso, estatura, circunferência do braço, prega cutânea tricipital e área muscular do braço, com a bioimpedância em crianças e adolescentes submetidos a tratamento quimioterápico. Tais pesquisadores concluíram que os parâmetros antropométricos apresentaram boa correlação entre si e com valores obtidos por bioimpedância. Segundo eles, a bioimpedância parece ser um método acurado para avaliação nutricional e aferição de compartimentos corporais em pacientes pediátricos portadores de neoplasias.

Lagares et al (2013) descreveram a prevalência de excesso de peso em mulheres diagnosticadas com câncer de mama submetidas à hormonioterapia com tamoxifeno. Foram coletadas informações sobre o estado nutricional utilizando parâmetros antropométricos como IMC, CC e porcentagem de gordura corporal por bioimpedância. Houve prevalência de sobrepeso e obesidade nas mulheres avaliadas em hormoninoterapia com tamoxifeno. Assim, os pacientes, especialmente aqueles que já tiveram história de sobrepeso, devem receber conselhos nutricionais desde o início da hormoninoterapia que promove a manutenção do peso saudável e devem ser encorajados a participar de programas de mudanças de estilo de vida.

Gupta et al. (2008) investigaram a associação entre o ângulo de fase derivado da Bioimpedância e Avaliação Global Subjetiva (AGS) no câncer colorretal avançado (estádios III e IV). Os pacientes foram classificados como bem nutridos ou desnutridos usando o AGS. A bioimpedância foi realizada em todos os pacientes e o ângulo de fase foi calculado. A correlação entre o ângulo de fase e a AGS foi realiza através do coeficiente de correlação de Spearman. Curvas características de operação do receptor foram estimadas usando o método não-paramétrico para determinar os níveis de corte ótimos de ângulo de fase. Os pesquisadores sugeriram que o ângulo de fase da bioimpedância é um indicador nutricional potencial no câncer colorretal avançado. Pesquisas adicionais são necessárias para elucidar os níveis de corte ótimos de ângulo de fase que podem ser incorporados na clínica de oncologia para melhor avaliação e manejo nutricional.

É sabido que várias ferramentas são usadas para determinar a caquexia, 
tais como: perda de peso, índice de massa corporal, rastreamento nutricional e composição corporal (Bioimpedância), ferramentas de imagem (absorciometria de raios $\mathrm{X}$ de energia dupla, tomografia computadorizada e ressonância magnética) e combinação de avaliação com marcadores inflamatórios. Schiessel (2013) mostraram novas definições de caquexia e ferramentas que determinam a caquexia em câncer. $\mathrm{O}$ mesmo considerou clinicamente importante a perda de peso (variando de $5 \%$ a $20 \%$ ou mais) e a correlacionou com o aumento da morbidade e mortalidade. Para ele, a perda de peso é o principal sintoma relatado por pacientes com câncer levando a caquexia e é muito importante para a progressão da doença, portanto, os médicos devem estar atentos a esta queixa.

Ao perceber que a caquexia reumatoide e a obesidade central eram comuns em pacientes com artrite reumatoide, Elkan et al. (2009) avaliou a composição corporal, o estado nutricional de pacientes com artrite reumatoide e a viabilidade da bioimpedância para detectar caquexia reumatoide. Os dados obtidos foram referentes ao Índice de Massa Corporal (IMC), Circunferência da Cintura (CC), Absorciometria de Raios-X de dupla energia de corpo inteiro (AXD), Bioimpedância e Miniavaliação Nutricional. Nesse estudo, foi possível concluir que a IMC e a MNA não poderiam relacionar a caquexia reumatoide e a obesidade central com a artrite reumatoide. Entretanto, houve um bom acordo relativo entre AXD e Bioimpedância, mas os limites de concordância foram amplos, podendo restringir a utilidade da Bioimpedância na prática clínica.

Silva et al. (2010) avaliaram o estado nutricional de pacientes com doença inflamatória intestinal em diferentes estágios de atividade inflamatória, por meio de dados antropométricos (aferição de peso, altura, circunferência do braço e prega cutânea do tríceps) e tiveram sua composição corporal determinada por bioimpedância. Para determinação de atividade inflamatória da doença foram utilizados os níveis séricos de proteína $\mathrm{C}$ reativa e o índice de Harvey e Bradshaw. Os autores concluíram que houve maior número de pacientes com excesso de peso e de gordura corporal, sendo que esses pacientes foram mais sintomáticos e apresentaram maiores valores de proteína $\mathrm{C}$ reativa.

\section{Patologias Neurológicas e pacientes ci- rúrgicos}

Simony et al. (2014) avaliaram o estado nutricional de crianças, jovens e adultos, os quais eram pacientes neurológicos em atendimento fisioterapêutico. 
Foram coletados dados sobre as variáveis antropométricas (peso, altura, circunferência da cintura ou abdominal), classificação do estado nutricional e o exame de bioimpedância (gordura corporal, massa magra e taxa metabólica basal) com aparelho TANITA ${ }^{\circledR}$ modelo TBF - 305. Dentre os sintomas gastrintestinais mais presentes, se destacaram a gastrite e o refluxo gastroesofágico. A maior parte dos pacientes adultos apresentou: eutrofia, seguida de obesidade e de sobrepeso. Nas crianças e jovens a prevalência de desnutrição foi de $20 \%$. A porcentagem de massa corporal foi em média de 24,2\%. Percebeu-se comprometimentos antropométricos e nutricionais importantes, os quais necessitam de acompanhamento sistemático para educação em saúde.

Oliveira e Frangella (2010) avaliaram e compararam a efetividade de diferentes métodos utilizados para avaliação nutricional de pacientes ambulatoriais acometidos por acidente vascular encefálico (AVC) isquêmico ou hemorrágico e hemiplégicos. Neste estudo, a força de preensão palmar e espessura do músculo adutor do polegar foram bem correlacionadas com as mensurações determinadas pela antropometria e pela bioimpedância (aparelho Biodynamics ${ }^{\circledR}$, modelo 310 ). Os resultados apontam a aplicabilidade da dinamometria e da espessura do músculo adutor do polegar na prática clínica como métodos de avaliação nutricional para essa população, especialmente idosos, pois detectam alterações funcionais não captadas por outros parâmetros em curto prazo, sendo importantes para identificação precoce do risco nutricional.

Pucci, Fontes e Poggetti (2008) avaliaram um esquema de realimentação utilizado em pacientes após jejum prolongado voluntário. A coleta dos dados antropométricos, laboratoriais, clínicos e dietéticos foi obtida do prontuário, $\mathrm{o}$ qual continha registrados da equipe multiprofissional do hospital, obteve-se também resultados da bioimpedância, como: peso diário, índice de massa corpórea (IMC), medidas da prega cutânea tricipital (PCT) e circunferência do braço (CB). No $32^{\circ}$ dia do jejum e no $8^{\circ}$ dia da realimentação constam registros da avaliação da composição corpórea determinada por bioimpedância (aparelho Biodynamics ${ }^{\circledR}$, modelo 310). O esquema de realimentação utilizado promoveu resultado global adequado, mas requer redução do aporte energético e definição de momento mais adequado para reintrodução de lactose e da dieta normolipídica.

Scheunemann et al. (2011) avaliaram a concordância e associação entre 
o ângulo de fase e os parâmetros do estado nutricional de pacientes cirúrgicos. A bioimpedância tetrapolar foi realizada utilizando um aparelho portátil calibrado (Biodynamics ${ }^{\circledR}$, modelo 310). O menor valor de concordância foi encontrado entre no ângulo de fase padronizado (AFP) e índice de massa corpórea (IMC). Esse resultado deve-se provavelmente aos elementos da bioimpedância, como ângulo de fase, e marcadores antropométricos, como o IMC, que expressam diferentes aspectos e estágios de deficiência nutricional. O AFP apresentou fraca concordância com os métodos de avaliação nutricional, assim como baixa especificidade, não podendo ser recomendado como marcador de estado nutricional, apesar de os menores valores de AFP terem sido encontrados em pacientes desnutridos.

\section{Distúrbio no equilíbrio de fluidos}

Identificar determinantes do estado de hidratação de pacientes em diálise peritoneal crônica e investigar os efeitos da sobrecarga líquida sobre o estado nutricional

Antunes et al. (2011) ao realizar experimentos com a finalidade de identificar determinantes do estado de hidratação de pacientes em diálise peritoneal crônica e investigar os efeitos da sobrecarga líquida sobre o estado nutricional percebeu que, pacientes com maior volume urinário e em modalidade dialítica automatizada apresentaram melhor estado de hidratação, e pacientes com maior sobrecarga líquida, comparados àqueles com menor sobrecarga, apresentaram menor ângulo de fase. Mediante os resultados encontrados sugeriu que os níveis reduzidos de albumina e ângulo de fase nos pacientes com maior sobrecarga líquida não correspondem ao pior estado nutricional. Para ele, o diagnóstico nutricional em vigência de sobrecarga líquida, deve-se considerar o conjunto de variáveis obtidas por diversos métodos, buscando relacioná-los e interpretá-los de maneira abrangente, possibilitando um diagnóstico nutricional fidedigno.

Berbel et al. (2011) realizaram uma avaliação nutricional de pacientes com lesão renal aguda para identificar a relação entre os marcadores nutricionais e seus resultados. Essa avaliação foi baseada na Avaliação Global Subjetiva (AGS), antropometria, análise de bioimpedância e nos parâmetros bioquímicos, e foram realizados diariamente ou semanalmente desde a primeira avaliação de nefrologia até recuperação da função renal ou morte do paciente. Concluiu que, uma avaliação nutricional em pacientes com lesão renal aguda parece identificar marcadores nutricionais que estão asso- 
ciados ao desfecho. Neste estudo, os riscos de morte em pacientes com lesão renal aguda foram associados a baixa ingestão calórica, a maior concentração de proteína $\mathrm{C}$ reativa, a presença de edema, menor resistência medida durante uma análise de bioimpedância e menor balanço de nitrogênio.

Vannini et al. (2009) avaliaram as associações clínicas, laboratoriais, demográficas, e marcadores nutricionais com estado inflamatório e desnutrição em pacientes com hemodiálise. O ângulo de fase foi positivamente associado com hematócrito, contagem de linfócitos totais e creatinina sérica, e foi negativamente associado com a idade. Observouse associação negativa e independente entre a porcentagem de músculo-massa (obtida da bioimpedância) e a inflamação. Esses resultados sugerem que o estado inflamatório induz a depleção da massa muscular, enquanto o alto IMC está associado com diabetes e menor pressão arterial diastólica, sendo um fator de risco cardiovascular reconhecido em pacientes urêmicos. $\mathrm{O}$ ângulo de fase e o SGA foram associados aos marcadores nutricionais tradicionais, reforçando sua validade para pacientes com hemodiálise.

Llames, Baldomero e Rodota (2013) descreveram valores de referência de ângulo fase (AF) de acordo com diferentes condições clínicas. Estudos revelam que o AF é o parâmetro da bioimpedância (BIA) bem estabelecido para o diagnóstico e prognóstico clínico de desnutrição, ambos associados com alterações na integridade da membrana celular e distúrbios no equilíbrio de fluidos. O AF expressa alterações na quantidade e qualidade da massa de tecido mole (ou seja, a permeabilidade celular e a membrana de hidratação). Diversos ensaios clínicos sugerem o AF como sendo um marcador de prognóstico útil em condições clínicas, tais como: cirrose hepática, câncer de mama, cólon, pâncreas e pulmão, como também em pacientes portadores de HIV.

Saxena, Sharma e Gupta (2016) avaliaram o efeito da função do enxerto sobre o estado nutricional usando bioimpedância (BIA) em pacientes transplantados renais com boa função do enxerto. Os parâmetros da bioimpedância avaliados incluído na fase ângulo foram: peso seco, água total do corpo, água extracelular, água intracelular, massa livre de gordura, massa gorda, a massa celular corporal, massa muscular, potássio corporal total, cálcio total do corpo e glicogênio, fluido do plasma, fluido intersticial e sólidos extracelulares. Escores Subjetivos de Avaliação Global (SAG) mostraram que os pacientes transplanta- 
dos tinham um estado nutricional normal, mas quando comparados com indivíduos saudáveis como avaliados pelo BIA, houve diferenças significativas na massa livre de gordura, massa gorda e massa celular corporal. Em conclusão, a BIA foi mais sensível para avaliar a depleção nutricional do que o SAG em pacientes transplantados com borderline.

Maicá e Schweigert (2008) contribuiram para a análise e recomendação de métodos eficazes, passíveis de utilização e fidedignos do ponto de vista da interpretação no contexto do paciente grave. Para eles um parâmetro isolado não caracteriza a condição nutricional do indivíduo, sendo necessário empregar

\section{CONSIDERAÇÕES FINAIS}

Atualmente é necessário um estudo minucioso, das publicações nacionais e internacionais para iniciar qualquer pesquisa seja ela original ou secundária, disponíveis nas bases de dados das bibliotecas virtuais, a respeito do tema pretendido, que garantam o download de publicações científicas, do tipo: artigos científicos, teses, dissertações, monografias, resumos de eventos científicos, livros etc. Contudo, o pesquisador necessita da habilidade de manusear terminologias (descritores) para tornar a busca da informação científica efetiva. Na área uma associação de vários indicadores para melhorar a precisão e a acurácia do diagnóstico nutricional. Os mesmos acreditam que os métodos se tornam limitados, devido características inerentes ao paciente crítico. No caso da bioimpedância, a aplicação e validade do método, para pacientes hospitalizados não foi completamente estudada para todas as situações clínicas. As limitações desse método estão relacionadas principalmente a fatores que alteram o estado de hidratação. Em paciente com alteração na distribuição hídrica (cardíacos, hepatopatas e nefropatas), a bioimpedância é indicada para acompanhar a evolução e não para avaliar o estado nutricional.

da saúde, essas terminologias são conhecidas como descritores em ciências da saúde e para obter tais vocábulos estruturados é preciso acessar os portais DeCS, Mesh e/ou Thesaurus.

No presente estudo foi obtido um levantamento de publicações científicas sobre avaliação nutricional por meio da bioimpedância na BVS usando descritores livre, estruturado e seus sinônimos. Os conteúdos dispostos em cada capítulo dessa monografia permitiu alcançar os objetivos estabelecidos nessa pesquisa.

Os portais DeCS, Mesh e BVS tiveram participação crucial na construção das informações citadas e discutidas, 
sendo eles considerados ferramentas essenciais do objeto de estudo. No geral, a BVS atendeu as principais necessidades da pesquisa, contemplando textos científicos sobre "Bioimpedância aplicada na avaliação nutricional" a nível mundial, em três idiomas, garantindo o acesso a publicações de variados periódicos.

O descritor livre bioimpedância obteve maior publicação escrita em português, quando comparado com os demais descritores. Esse descritor não é codificado, não possui identificador único, justificando seu baixo potencial indexador. Contudo, os resultados apontam a necessidade de inserí-lo no cadastro de registro $\mathrm{DeCS} / \mathrm{MeSH}$, uma vez que o número total de publicações foi bem inferior e um quantitativo de publicações disponíveis muito próximo aos demais descritores analisados, caracterizando uma importante terminologia.

A busca do tipo $I A H x$, presente na BVS, ofereceu recursos para o refinamento dos resultados de varredura por meio dos filtros (clusters), de maneira que a produção científica pôde ser estimada nas ciências da saúde a partir de metadados inseridos nas bases de dados, tais como: texto completo, coleções, bases de dados, assunto principal, tipo de estudo, aspecto clínico, limite, país/re- gião como assunto, idioma, ano de publicação, tipo de documento, revista, assunto da revista e país de afiliação.

Os filtros (metadados) devem ser atualizados com frequência e o quantitativo dos dados nos filtros deve corresponder ao número total de publicações citadas para que pesquisadores possam utilizar essas informações e compará-las com os descritores usados. Na BVS foi comum encontrar incompatibilidade no somatório dos dados distribuídos nos filtros, que não correspondiam com a totalidade de publicações citadas, além da ausência de alguns filtros nos resultados de alguns descritores pesquisados. Esses tipos de falhas precisam ser corrigidas para evitar a publicação de documentos científicos, com informações errôneas, que abordem dados extraídos dessa biblioteca virtual.

Metadados ou informações adicionais podem ser inseridos na BVS, como: nome do autor principal, instituição onde a pesquisa foi realizada, Qualis, fator de impacto, tempo de submissão/aprovação/publicação, gratuidade ou não da publicação, tipo de publicação (impressa ou online), tipo de metodologia adotada, objeto de estudo, normas de publicação, estilo de referência bibliográfica, valor de aquisição do documento científico e valor da publicação, contato 
do pesquisador, link do currículo do autor principal, tipo de estudo (primário ou secundário). Isso porque essas informações garantem uma autonomia ao pesquisador e melhor direcionamento na aquisição e publicação de documentos científicos.

Várias são as estratégias de busca utilizadas para se obter textos científicos que abordem a terminologia pesquisada. Nesse trabalho, as estratégias utilizadas foram: colocar aspas nos vocabulários para busca do termo exato e usar descritores estruturados (DeCS e Mesh) e livre. Com as estratégias de busca utilizadas nessa pesquisa foi possível obter êxito no levantamento de publicações científicas sobre o tema proposto, de modo a facilitar o trabalho do pesquisador em relação à tomada de decisão, poupar tempo, garantir uma revisão completa a respeito do assunto.

Estudos como esse, permitem uma discussão profunda sobre bioimpedância na avaliação nutricional garantindo mais informações integradas para os multiprofissionais que realizam esse tipo de exame ou pretendem usar esse método em seus consultórios, clínicas, estúdios e academias. Pois, a análise da composição corporal é parte essencial dos programas de emagrecimento, de condicionamento físico, além de contribuir na profilaxia e tratamento de doenças crônicas como diabetes, hipertensão arterial, dislipidemias e cardiopatias. Os resultados, desse estudo, irão subsidiar futuras pesquisas que tratem da temática "Bioimpedância na avaliação nutricional".

Enfatiza-se, entretanto, a necessidade de investigar os procedimentos clínicos realizados por meio da bioimpedância, acessar as publicações cientifica disponíveis sobre essa temática e integrar os achados desses estudos, no sentido de comparar os métodos clínicos atualmente aplicados na avaliação da composição corporal e estado nutricional para obtenção do melhor método que vise acessibilidade, custo-benefício e aceitação do paciente.

Por fim, o pesquisador, que acerta na escolha do (s) descritor(es), da(s) biblioteca(s) virtual(is) e de seu(s) filtro(s), tem êxito no levantamento de publicações científicas sobre o tema pesquisado, isto porque facilita seu trabalho quanto a tomada de decisão, poupa tempo e garante uma varredura completa. 


\section{REFERÊNCIA BIBLIOGRÁFICA}

ACUÑA, K.; CRUZ, T. Avaliação do Estado Nutricional de Adultos e Idosos e Situação Nutricional da População Brasileira. Arq Bras Endocrinol Metab, v. 48, n. 3, p. 345-361, 2004.

ANTUNES, A. de A. et al. Relevância do estado de hidratação na interpretação de parâmetros nutricionais. Rev. Nut., v. 24, n. 1, p. 99-107, 2011.

AURÉLIO, R. et al. Arterial stiffness is associated with low skeletal muscle mass in Japanese community-dwelling older adults. Geriatr Gerontol Int, v. 14, n. 1, p. 109-114, doi:10.1111/ggi.12206, 2014.

BALBINO, V.; SILVA, O. de C. e. Nutritional assessment of cir rhotic cirrhotic $\mathrm{w}$ a ppr oac $\mathrm{h}$ based on pa tients : A new patients : new appr pproac oach electrical bioimpedance. Medicina (Ribeirão Preto), v. 45, n. 1, p. 1-4, 2012.

BARDIN, L. Análise de conteúdo. $3^{\mathrm{a}}$ ed. Lisboa: Edições 70; 2004.

BENTON, M.J.; G.W. STORRS. Testing the quality of the fossil record: paleontological knowledge is improving. Geology, vol. 22, p.111-114, 1994.

BERBEL, M. N. et al. Nutritional parameters are associated with mortality in acute kidney injury. CLINICS, v. 69 , n. 7, p. 476-482, 2011.

BORGES, V. C. et al. Long-term nutritional assessment of patients with severe short bowel syndrome managed with home enteral nutrition and oral intake. Nutr Hosp. 2011;26(4):834-842, v. 26 , n. 4 , p. $834-842$, doi: 10.3305/nh.2011.26.4.5153, 2011.

BOTELHO, L. L. R.; CUNHA, C.C. de A.; MACEDO, M. O método da revisão integrativa nos estudos organizacionais.
Gestão e sociedade, v.5, n.11, p.121136, 2011. BVS, 2017. Disponível em: $<$ http://bvsalud.org/>. Acesso em: 01/05/2017.

CARRARO, J. de L.; SCHWARTZ, R.; BEHLING, E. B. Comparação do estado nutricional obtido através de antropometria e bioimpedância em crianças e adolescentes submetidos a tratamento quimioterápico em um hospital no sul do brasil. Revista HCPA, v. 32, n. 1, p. 35-41, 2012.

COCETTI M., CASTILHO S. D., BARROS FILHO AA. Dobras cutâneas e bioimpedância elétrica perna-perna na avaliação da composição corporal de crianças. Ver Nutr, vol. 22, n. 4, p.52736, 2009.

DECS, 2017. Disponível em: < http://decs.bvs.br/cgibin/wxis1660.exe/decsserver/?IsisScript=../cgi-bin/decsserver/decsserver.xis\&interface_language $=$ p\&previous_page $=$ homepage\&previous_task $=$ NULL \& task $=$ start $>$. 01/03/2016. Acesso em: 01/05/2017.

EICKEMBERG, M. et al., Bioimpedância elétrica e sua aplicação em avaliação nutricional. Rev Nutr, vol. 24, n. 6, p. 883-93, 2011.

ELKAN, A. et al. Rheumatoid cachexia, central obesity and malnutrition in patients with low-active rheumatoid arthritis : feasibility of anthropometry, Mini Nutritional Assessment and body composition techniques. Eur J Nutr (2009), v. 48, p. 315-322, 2009.

FERNANDES, S. A. et al. NUTRITIONAL ASSESSMENT IN PATIENTS WITH CIRRHOSIS. Arq Gastroenterol, v. 49, n. 1, p. 19-27, 2012.

FUJIOKA Y. How do we treat body fat percentages determined by bioelectrical 
impedance analysis? Circ J, vol. 76, n. 10, p. 2335-6, 2012;

GANONG, L. H. Integrative review of nursing research. Res Nurs Health, v. 10, n.1, p.1-11, 1987.

GUEDES, D. P. Procedimentos clínicos utilizados para análise da composição corporal. Rev Bras Cineantropom Desempenho Hum, vol. 15, n. 1, p. 113129, 2013.

GUPTA, D. et al. The relationship between bioelectrical impedance phase angle and subjective global assessment in advanced colorectal cancer. Nutrition Journal, v. 7, n. 19, p. 1-6, 2008.

LAGARES, E. B. et al. Excesso de Peso em Mulheres com Diagnóstico de Câncer de Mama em Hormonioterapia com Tamoxifeno Overweight Breast Cancer Diagnosed Women undergoing Hormoninotherapy with Tamoxifeno. Revista Brasileira de Cancerologia, v. 59, n. 2, p. 201-210, 2013.

LIMA, L. D. de; MORAES, C. M. B. de; KIRSTEN, V. R. Dismorfia Muscular e o Uso de Suplementos Ergogênicos em Desportistas. Rev Bras Med Esporte, v. d, n. 4, p. 427-430, 2010.

LLAMES, L.; BALDOMERO, V.; RODOTA, M. L. I. L. P. Valores del ángulo de fase por bioimpedancia eléctric; estado nutricional y valor pronóstico. Nutr Hosp., v. 28, n. 2, p. 286-295, 2013.

MACHADO, R. S. P.; COELHO, M. A. S. C.; COELHO, K. S. C. Percentual de gordura corporal em idosos: comparação entre os métodos de estimativa pela área adiposa do braço, pela dobra cutânea tricipital e por bioimpedância tetrapolar. Rev. Bras. Geriatr. Gerontol., v. 13, n. 1, p. 17-28, 2006.
MAICÁ, A. O.; SCHWEIGERT, I. D. Avaliação nutricional em pacientes graves. Rev Bras Ter Intensiva. v. 20, n. 3 , p. 286-295, 2008.

MELLO, M. T. et al. Avaliação da composição corporal em adolescentes obesos: o uso de dois diferentes métodos. Rev Bras Med Esporte, vol. 11, n. 5, p. 267-70, 2005.

MESH, 2016.

$<$ http://www.ncbi.nlm.nih.gov/mesh/?te $\mathrm{rm}=$ mesh $>$. Acesso em: 05/03/2017.

MORAIS, P. A. de O. et al. Métodos indiretos para mensuração da gordura corporal em adolescentes escolares. Rev Bras Promoc Saude, vol. 26, n.3, p. 412-418, 2013.

OLIVEIRA, D. R.; FRANGELLA, V. S. Músculo adutor do polegar e força de preensão palmar: potenciais métodos de avaliação nutricional em pacientes ambulatoriais com acidente vascular encefálico. Einstein, v. 8, n. 4, p. 467-472, 2010 .

PACKER, A. L.; TARDELLI, A. O.; CASTRO, R. C. F. A distribuição do conhecimento científico público em informação, comunicação e informática em saúde indexado nas bases de dados MEDLINE e LILACS Public scientific knowledge distribution in health information, communication and information technology ind. Ciência \& Saúde Coletiva, v. 12, n. 3, p. 587-599, 2007.

PELLIZZON, R. de F. Pesquisa na área da saúde. 1. Base de dados DeCS (Descritores em Ciências da Saúde). Acta Cirúrgica Brasileira, v. 19, n. 2, p. 153-163, 2004.

PUCCI, N. D.; FONTES, B.; POGGETTI, R. S. Avaliação de um esquema de realimentação utilizado após 43 dias de jejum voluntário Evaluation of a refeeding plan employed after 43 
days of voluntary fasting. Rev. Nutr, v. 21, n. 5, p. 503-512, 2008.

SANT'ANNA, M. de S. L.; PRIORE, S. E.; FRANCESCHINI, S. do C. C. Métodos de avaliação da composição corporal em crianças. Rev Paul Pediatr, v. 27, n. 3, p. 315-321, 2009.

SAXENA, A.; SHARMA, R. K.; GUPTA, A. Graft Function and Nutritional Parameters in Stable Postrenal Transplant Patients. Saudi J Kidney Dis Transpl, v. 27, n. 2, p. 356-361, 2016.

SCHEUNEMANN, L. et al. Agreement and association between the phase angle and parameters. Nutr Hosp., v. 26, n. 3, p. $480-487,2011$.

SCHIESSEL, D. L. Definitions and Tools to Assessing Cancer Cachexia : a Review Definições e Ferramentas para Avaliar a Caquexia no Câncer : uma Revisão. Revista Brasileira de Cancerologia, v. 59, n. 4, p. 583-591, 2013.

SCHIPER, L. et al. Hip fracture prognosis: could bioimpedance be an alternative to conventional nutritional assessment? Nutr Hosp. v. 26, n. 4, p. 904-906, 2011.

SCHMOELLER, R. et al. Cargas de trabalho e condições de trabalho da enfermagem: revisão integrativa. Rev Gaúcha Enferm. v. 32, n. 2, p. 368-377, 2011.

SILVA, A. F. dá et al. relação entre estado nutricional e atividade inflamatória em pacientes com doença inflamatória intestinal. ABCD Arq Bras Cir Dig, v. 23, n. 3, p. 154-158, 2010.
SILVA, E. L. Da. Metodologia da Pesquisa e Elaboração de Dissertação. 4. ed. Florianópolis: UFSC, 2005. p. 138.

SIMONY, R. F. et al. Caracterização do estado nutricional dos pacientes nutritional status of neurological patients with reduced mobility. Journal of Human Growth and Development, v. 24, n. 1, p. 42-48, 2014.

THESAURUS. Disponível em: $<$ http://pergamum.inep.gov.br/pergamum/biblioteca/pesquisa_thesauro.php? resolution2 $=1024 \_1>$. Acesso em: 01/05/2017.

TREMESCHIN, M. H. et al. Nutritional assessment and lipid profile in HIV-infected children and adolescents treated with highly active antiretroviral therapy Avaliação nutricional e do perfil lipídico em crianças e adolescentes infectadas pelo HIV tratadas com terapia antirretrovi. Revista da Sociedade Brasileira de Medicina Tropica, v. 44, n. 3, p. 274-281, 2011.

VANNINI, F. D. et al. Associations between nutritional markers and inflammation in hemodialysis patients. Int Urol Nephrol, v. 41, p. 1003-1009, 2009.

VULCANO, D. S. B.; CARVALHAES, M. A. de B. L.; BAKONYI NETO, A. Evaluation of nutritional indicators and body composition in patients with advanced liver disease enrolled for liver transplantation 1. Acta Cirúrgica Brasileira, v. 28, n. 10, p. 733-739, 2013. 